\title{
Variational Iteration Algorithm-I with an Auxiliary Parameter for Solving Fokker-Planck Equation
}

\author{
Hijaz Ahmad \\ Department of Basic Sciences, University of Engineering and Technology, Peshawar, Pakistan \\ e-mail: hijaz555@gmail.com
}

\begin{abstract}
In this paper, variational iteration algorithm-I with an auxiliary parameter is implemented to investigate Fokker-Planck equations. To show the accuracy and reliability of the technique comparisons are made between the variational iteration algorithm-I with an auxiliary parameter and classic variational iteration algorithm-I. The comparison shows that variational iteration algorithm-I with an auxiliary parameter is more powerful and suitable method for solving Fokker-Planck equations. Furthermore, the proposed algorithm can successfully be applied to a large class of nonlinear and linear problems.
\end{abstract}

\section{Introduction}

The aim of this work is to apply the variational iteration algorithm-I [1] with an auxiliary parameter for the analytical treatment of the Fokker-Planck equation. The method is able to provide analytical results for nonlinear and linear problems, in a direct way very conveniently. One of the main characteristics of this method is that approximate solution of great accuracy can be obtained by only a few iterations. This method has a simple procedure, acceptable results and above all, this method can successfully be applied to a large class of linear and nonlinear problems [2]-[6].

Received: March 11, 2019; Accepted: April 14, 2019

2010 Mathematics Subject Classification: 65-XX.

Keywords and phrases: Fokker-Planck equation, Kolmogorov equation, variational iteration algorithm-I, variational iteration algorithm-I with an auxiliary parameter.

Copyright (C) 2019 Hijaz Ahmad. This is an open access article distributed under the Creative Commons Attribution License, which permits unrestricted use, distribution, and reproduction in any medium, provided the original work is properly cited. 


\section{Variational Iteration Algorithm-I}

Consider a general differential equation

$$
L[u(x)]+N[u(x)]=c(x),
$$

the terms $L[u(x)]$ and $N[u(x)]$ represent the linear and nonlinear term respectively, while $c(x)$ is the inhomogeneous source term. Constructing a correction function for Eq. (1) as,

$$
u_{k+1}(x)=u_{k}(x)+\int_{0}^{x} \lambda(\eta)\left[L\left\{u_{k}(\eta)\right\}+N\left\{\widetilde{u_{k}(\eta)}\right\}-c(\eta)\right] d \eta
$$

where $\lambda$ is a parameter, which is not known and called the Lagrange multiplier [7].

Taking the variation $\delta$ on the one side as well as the other side of Eq. (2) with respect to $u_{k}(x)$,

$$
\delta u_{k+1}(x)=\delta u_{k}(x)+\delta \int_{0}^{x} \lambda(\eta)\left[L\left\{u_{k}(\eta)\right\}+N\left\{\widetilde{u_{k}(\eta)}\right\}-c(\eta)\right] d \eta
$$

where $\widetilde{u_{k}(\eta)}$ is considered as a restricted term which means $\overline{\delta u_{k}(\eta)}=0$.

Using optimality conditions, the value of Lagrange multiplier $\lambda(\eta)$ can be identified. An exact solution obtains when $k \rightarrow \infty$.

$$
u(x)=\lim _{k \rightarrow \infty} u_{k}(x)
$$

In short, the formula for equation (1) is,

$$
\left\{\begin{array}{l}
u_{0}(x) \text { is an appropriate initial approximation, } \\
u_{k+1}(x)=u_{k}(x)+\int_{0}^{x} \lambda(\eta)\left[L\left\{u_{k}(\eta)\right\}+N\left\{u_{k}(\eta)\right\}-c(\eta)\right] d \eta \\
k=0,1,2,3, \ldots
\end{array}\right.
$$

This technique is called VIA-I, which is a further development of the general Lagrange multiplier technique for solving nonlinear problems by Inokuti et al. [7]. Now this method [8]-[10] has been developed [11-12] to solve a lot of problems arise in various fields of sciences. 


\section{Insertion of an Auxiliary Parameter in Variational Iteration Algorithm-I}

In VIA-I, an auxiliary parameter $h$ can be inserted. The optimal choice of unknown $h$ improves the correctness, precision and effectiveness of the technique. After inserting $h$, equation (5) will become

$$
\left\{\begin{array}{l}
u_{0}(x) \text { is an appropriate initial approximation, } \\
u_{1}(x, h)=u_{0}(x)+h \int_{0}^{x} \lambda(\eta)\left[L\left\{u_{0}(\eta)\right\}+N\left\{u_{0}(\eta)\right\}-c(\eta)\right] d \eta, \\
u_{k+1}(x, h)=u_{k}(x, h)+h \int_{0}^{x} \lambda(\eta)\left[L\left\{u_{k}(\eta, h)\right\}+N\left\{u_{k}(\eta, h)\right\}-c(\eta, h)\right] d \eta, \\
k=1,2,3, \ldots
\end{array}\right.
$$

This technique is known as VIA-I with AP. Actually, this technique is simple, has a lesser size of calculation, not difficult to analyze and have the ability to approximate the solution precisely in solution domain of wide range.

\section{The Fokker-Planck Equation [2]}

In this section, the general form of Fokker-Planck equation which is also called forward Kolmogorov equation is

$$
\frac{\partial u}{\partial t}=\left[\frac{\partial}{\partial t} A(x)+\frac{\partial^{2}}{\partial x^{2}} B(x)\right] u(x, t),
$$

with conditions:

$$
u(x, 0)=f(x), \quad x \in \mathcal{R} .
$$

It is the equation for the motion of concentration field $u(x, t)$. The backward Kolmogorov equation can be written in the following form

$$
\frac{\partial u}{\partial t}=-\left[\frac{\partial}{\partial t} A(x, t)+\frac{\partial^{2}}{\partial x^{2}} B(x, t)\right] u(x, t) .
$$

Let the initial conditions,

$$
u(x, 0)=f(x), \quad x \in \mathcal{R}
$$

and

$$
A(x, t)=-(x+1),
$$




$$
B(x, t)=x^{2} e^{t}
$$

Then equation (8) becomes

$$
\frac{\partial u}{\partial t}=\left[\frac{\partial^{2}}{\partial x^{2}} x^{2} e^{t}-\frac{\partial}{\partial t}(x+1)\right] u(x, t) .
$$

First, we solve this example by VIA-I.

Constructing the correction function for equation (9) as,

$$
\begin{aligned}
u_{k+1}(x, t)= & u_{k}(x, t) \\
& +\int_{0}^{t} \lambda(\eta)\left\{\frac{\partial u_{k}(x, \eta)}{\partial \eta}+\frac{\partial u_{k}(x+\tilde{1})}{\partial \eta} u_{k}(x, \eta)-\frac{\partial^{2}\left(\tilde{x}^{2} e^{\eta}\right)}{\partial x^{2}} u_{k}(x, \eta)\right\} d \eta .
\end{aligned}
$$

Taking the variation $\delta$ on the one side as well as the other side with respect to $u_{k}(x, t)$

$$
\begin{aligned}
\delta u_{k+1}(x, t)= & \delta u_{k}(x, t) \\
& +\delta \int_{0}^{t} \lambda(\eta)\left\{\frac{\partial u_{k}(x, \eta)}{\partial \eta}+\frac{\partial u_{k}(x+\tilde{1})}{\partial \eta} u_{k}(x, \eta)-\frac{\partial^{2}\left(\tilde{x}^{2} e^{\eta}\right)}{\partial x^{2}} u_{k}(x, \eta)\right\} d \eta .
\end{aligned}
$$

Ignoring the restricted terms

$$
\begin{aligned}
\delta u_{k+1}(x, t) & =\delta u_{k}(x, t)+\delta \int_{0}^{t} \lambda(\eta)\left\{\frac{\partial u_{k}(x, \eta)}{\partial \eta}\right\} d \eta \\
& =\delta u_{k}(x, t)+\lambda(\eta) \delta u_{k}(x, t)-\int_{0}^{t} \lambda^{\prime}(\eta) \delta u_{k}(x, \eta) d \eta \\
& =(1+\lambda(\eta)) \delta u_{k}(x, t)-\int_{0}^{t} \lambda^{\prime}(\eta) \delta u_{k}(x, \eta) d \eta .
\end{aligned}
$$

The stationary conditions are:

$$
\begin{gathered}
\lambda^{\prime}(\eta)=0, \\
1+\lambda(\eta)=0,
\end{gathered}
$$

we get the value of $\lambda(\eta)$ which is $\lambda(\eta)=-1$. 
Using this value of $\lambda(\eta)$ in equation (10) results in the below iterative scheme:

$$
\begin{aligned}
u_{k+1}(x, t)= & u_{k}(x, t) \\
& -\int_{0}^{t}\left\{\frac{\partial u_{k}(x, \eta)}{\partial \eta}+\frac{\partial u_{k}(x+1)}{\partial \eta} u_{k}(x, \eta)-\frac{\partial^{2}\left(x^{2} e^{\eta}\right)}{\partial x^{2}} u_{k}(x, \eta)\right\} d \eta .
\end{aligned}
$$

starting with

$$
u_{0}(x, t)=x+1
$$

other approximations by using the scheme (11),

$$
\begin{aligned}
& u_{1}(x, t)=(t+1)(x+1), \\
& u_{2}(x, t)=\frac{(x+1)\left(\left(t^{2}+2 t+2\right)\right)}{2}, \\
& u_{3}(x, t)=\frac{(x+1)\left(\left(t^{3}+3 t^{2}+6 t+6\right)\right)}{6},
\end{aligned}
$$

we stop the procedure at $u_{10}(x, t)$. The absolute error of $u_{10}(x, t)$ in the solution domain $(x, t) \in[0,5] \times[0,1]$ can be seen in Figure 1 .

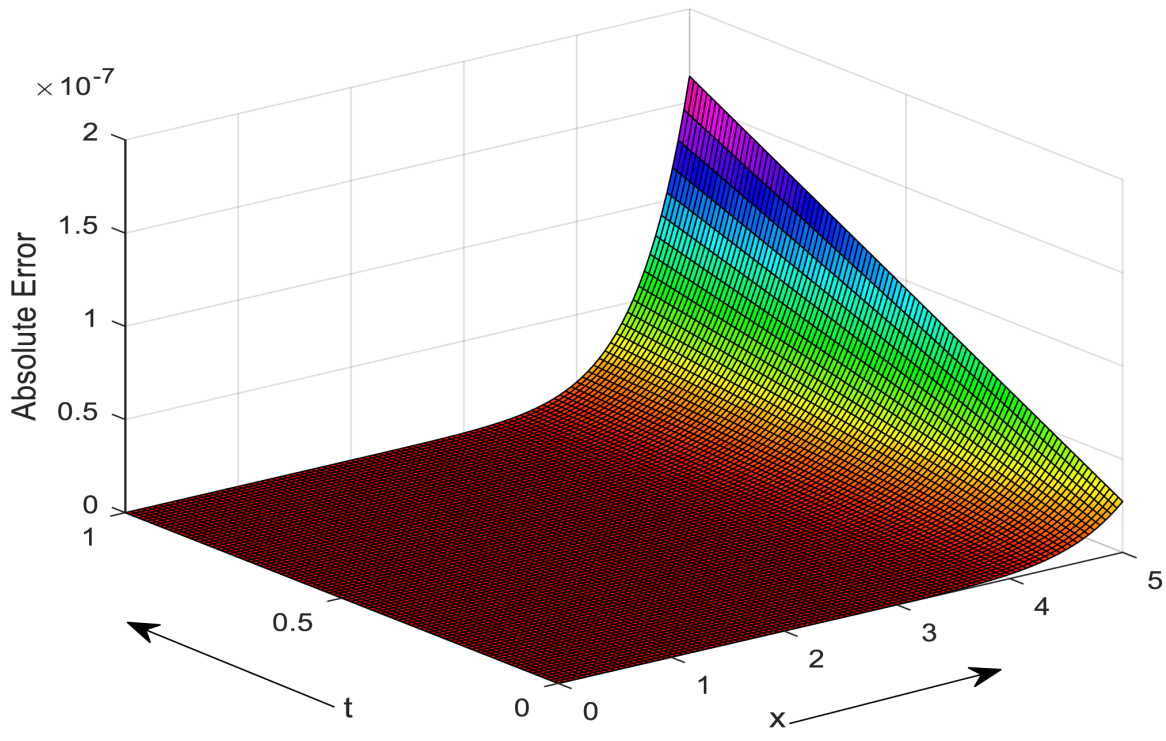

Figure 1. Absolute error betwixt the exact and approximate solutions by VIA-I. 
Now we want to solve this problem by VIA-I with AP.

Using VIA-I with AP, the recurrence relation for equation (9) is

$$
\begin{gathered}
u_{k+1}(x, t, h)=u_{k}(x, t, h)-h \int_{0}^{t}\left\{\frac{\partial u_{k}(x, \eta, h)}{\partial \eta}+\frac{\partial u_{k}(x+1)}{\partial \eta} u_{k}(x, \eta, h)\right. \\
\left.-\frac{\partial^{2}\left(x^{2} e^{\eta}\right)}{\partial x^{2}} u_{k}(x, \eta, h)\right\} d \eta .
\end{gathered}
$$

Starting with

$$
u_{0}(x, t)=x+1
$$

Other approximations can be get by using the recurrence relation (12),

$$
\begin{aligned}
& u_{1}(x, t, h)=(h t+1)(x+1) \\
& u_{2}(x, t, h)=(h t+1)(x+1)+\frac{h t(x+1)((h t-2 h+2))}{2} \\
& u_{3}(x, t, h)=\frac{(x+1)\left(h^{3} t^{3}-6 h^{3} t^{2}+6 h^{3} t+9 h^{2} t^{2}-18 h^{2} t+18 h t+6\right)}{6},
\end{aligned}
$$

we stop the procedure at $u_{10}(x, t, h)$.

The following residual function is defined

$$
r_{10}(x, t, h)=\frac{\partial u_{10}(x, t, h)}{\partial \eta}+\frac{\partial u_{10}(x+1)}{\partial \eta} u_{10}(x, t, h)-\frac{\partial^{2}\left(x^{2} e^{\eta}\right)}{\partial x^{2}} u_{10}(x, t, h) .
$$

The square of residual function for 10th-order approximation with respect to $h$ for $(x, t) \in[0,5] \times[0,1]$ is

$$
\frac{1}{(11)^{2}} \sum_{i=0}^{10} \sum_{j=0}^{10}\left(r_{10}\left(\frac{5 i}{10}, \frac{j}{10}, h\right)\right)^{2}
$$

The minimum value of above square residual function occurs at $h=1.02408718538499$. Using this value of $h$ in $u_{10}(x, t, h)$ in the solution domain $(x, t) \in[0,5] \times[0,1]$, error betwixt the exact and approximate solutions can be seen in Figure 2. 


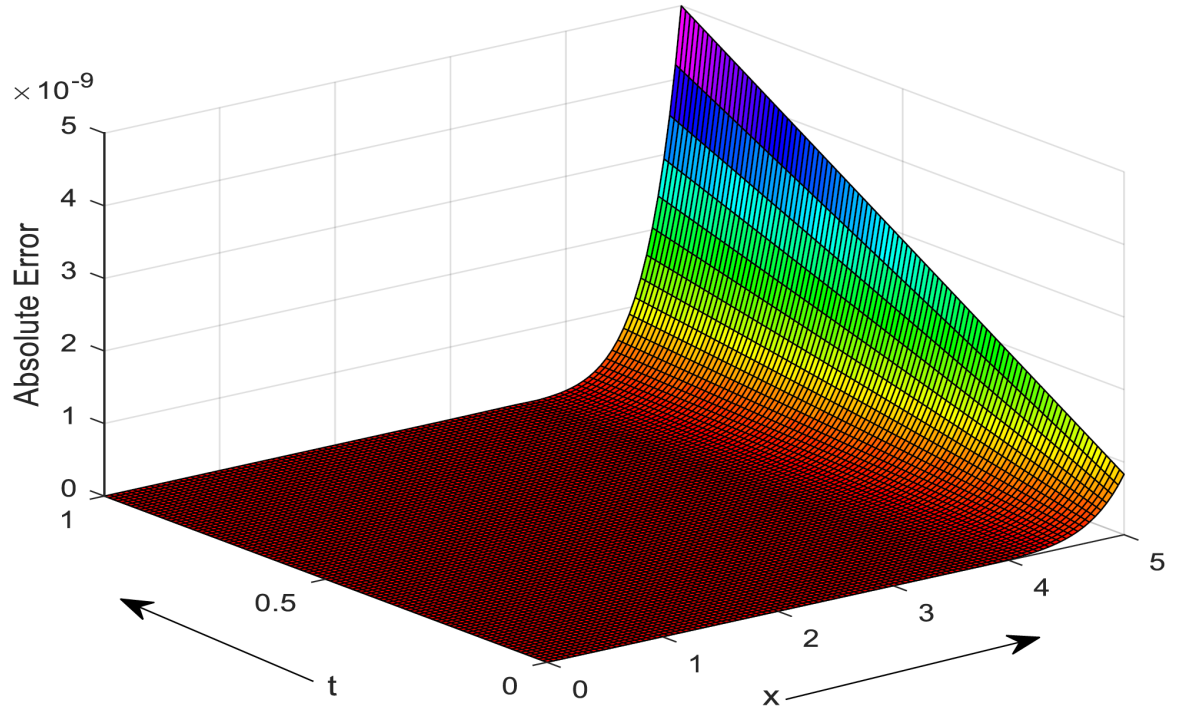

Figure 2. Absolute error betwixt the approximate and exact solutions by VIA-I with AP.

Comparing Figure 1 and Figure 2, it is clear that VIA-I with AP gives better results as compared to VIA-I. Numerical comparison betwixt the exact and approximate solutions of both methods is given in the table below.

Table 1. Comparison of absolute errors for 6th order approximation by VIA-I and VIA-I with AP.

\begin{tabular}{|c|c|c|c|}
\hline $\boldsymbol{x}$ & $\boldsymbol{t}$ & $\begin{array}{c}\text { Absolute Error in } \\
\text { VIA-I with AP }\end{array}$ & $\begin{array}{c}\text { Absolute Error in } \\
\text { VIA-I }\end{array}$ \\
\hline 0.5 & 0.1 & $3.553 \times 10^{-16}$ & $2.220 \times 10^{-16}$ \\
\hline 1.0 & 0.2 & $2.665 \times 10^{-15}$ & $1.332 \times 10^{-15}$ \\
\hline 1.5 & 0.3 & $2.398 \times 10^{-14}$ & $1.137 \times 10^{-13}$ \\
\hline 2.0 & 0.4 & $7.994 \times 10^{-15}$ & $3.261 \times 10^{-12}$ \\
\hline 2.5 & 0.5 & $6.750 \times 10^{-14}$ & $4.467 \times 10^{-11}$ \\
\hline 3.0 & 0.6 & $4.174 \times 10^{-13}$ & $3.826 \times 10^{-10}$ \\
\hline 3.5 & 0.7 & $3.608 \times 10^{-12}$ & $2.376 \times 10^{-09}$ \\
\hline 4.0 & 0.8 & $3.947 \times 10^{-11}$ & $1.152 \times 10^{-08}$ \\
\hline 4.5 & 0.9 & $6.701 \times 10^{-10}$ & $4.672 \times 10^{-08}$ \\
\hline 5.0 & 1.0 & $4.997 \times 10^{-09}$ & $1.639 \times 10^{-07}$ \\
\hline
\end{tabular}


The above table shows that VIA-I with AP is better for a large domain of $t$ as compared to VIA-I.

\section{Conclusions}

In this paper, variational iteration algorithm-I with an auxiliary parameter has been used in a way that accomplished the desired aim for solving Fokker-Planck equation. This work has made sure that the variational iteration algorithm-I with an auxiliary parameter offers noteworthy advantages in terms of its easy applicability, its computational success, and its adequacy to solve a wide class of differential equations. Graphical and numerical results reveal that this modification of variational iteration algorithm-I is suitable for all linear and nonlinear problems arise in physical sciences and engineering, superior to the variational iteration algorithm-I.

\section{References}

[1] J.-H. He, G.-C. Wu and F. Austin, The variational iteration method which should be followed, Nonlinear Sci. Lett. A 1(1) (2010), 1-30.

[2] J. Biazar, P. Gholamin and K. Hosseini, Variational iteration method for solving FokkerPlanck equation, J. Franklin Inst. 347(7) (2010), 1137-1147.

[3] M. Rafiq, H. Ahmad and S. T. Mohyud-Din, Variational iteration method with an auxiliary parameter for solving Volterra's population model, Nonlinear Sci. Lett. A 8(4) (2017), 389-396.

[4] H. Ahmad, Variational iteration method with an auxiliary parameter for solving differential equations of the fifth order, Nonlinear Sci. Lett. A 9(1) (2018), 27-35.

[5] M. M. Hosseini, S. T. Mohyud-Din, H. Ghaneai and M. Usman, Auxiliary parameter in the variational iteration method and its optimal determination, Int. J. Nonlinear Sci. Numer. Simul. 11(7) (2010), 495-502.

[6] J.-H. He, Variational iteration method - a kind of non-linear analytical technique: some examples, Int. J. Non. Linear. Mech. 34(4) (1999), 699-708.

[7] M. Inokuti, H. Sekine and T. Mura, General Use of the Lagrange Multiplier in Nonlinear Mathematical Physics, Oxford: Pergamon Press, 1978.

[8] J.-H. He, Variational approach to the Thomas-Fermi equation, Appl. Math. Comput. 143(2-3) (2003), 533-535. 
[9] J.-H. He, Variational iteration method-Some recent results and new interpretations, J. Comput. Appl. Math. 207(1) (2007), 3-17.

[10] J.-H. He and X.-H. Wu, Variational iteration method: new development and applications, Comput. Math. Appl. 54(7-8) (2007), 881-894.

[11] H. Ahmad, Auxiliary parameter in the variational iteration algorithm-II and its optimal determination, Nonlinear Sci. Lett. A 9(1) (2018), 62-72.

[12] H. Ahmad, Variational iteration algorithm-I with an auxiliary parameter for wave-like vibration equations, Journal of Low Frequency Noise, Vibration and Active Control (2019), to appear. https://doi.org/10.1177/1461348418823126 